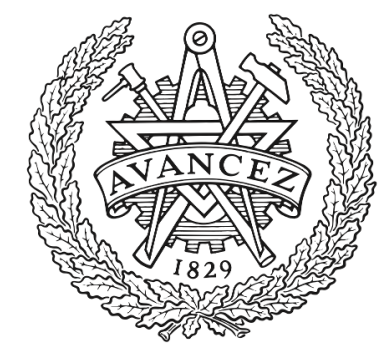

CHALMERS

UNIVERSITY OF TECHNOLOGY

\title{
Low Loss Silicon-Rich Silicon Nitride for Nonlinear Optics
}

Downloaded from: https://research.chalmers.se, 2023-04-26 10:52 UTC

Citation for the original published paper (version of record):

Ye, Z., Fülöp, A., Helgason, Ò. et al (2018). Low Loss Silicon-Rich Silicon Nitride for Nonlinear Optics. 2018 Conference on Lasers and Electro-Optics, CLEO 2018 - Proceedings. http://dx.doi.org/10.1364/CLEO_SI.2018.STu3F.1

N.B. When citing this work, cite the original published paper. 


\title{
Low Loss Silicon-Rich Silicon Nitride for Nonlinear Optics
}

\author{
Zhichao Ye,* Attila Fülöp, Óskar Bjarki Helgason, Peter A. Andrekson, and Victor Torres-Company \\ Photonics Laboratory, Department of Microtechnology and Nanoscience, Chalmers University of Technology,SE-41296 \\ zhichao@chalmers.se
}

\begin{abstract}
We demonstrate low loss $(\sim 0.4 \mathrm{~dB} / \mathrm{cm})$ silicon-rich silicon nitride waveguides and high $\mathrm{Q}$ microresonators $\left(\mathrm{Q}_{\mathrm{i}} \sim 10^{6}\right)$ featuring broadband anomalous dispersion. Microresonator combs are generated for the first time in this emerging material platform.

OCIS codes: (220.4000) Optical design and fabrication: Microstructure fabrication; (190.4360) Nonlinear optics: Nonlinear optics, devices; (230.5750) Optical devices: Resonators
\end{abstract}

\section{Introduction}

Stoichiometric silicon nitride $\left(\mathrm{Si}_{3} \mathrm{~N}_{4}\right)$ has attracted significant attention for on-chip nonlinear optics because of its CMOS compatibility [1], low optical material loss and high nonlinearity [2]. The stoichiometry of the material composition can be precisely controlled [3]. In particular, a slight increase in silicon with respect to the stoichiometric composition yields very promising advantages for nonlinear optics. It shows increased second- [4] and third-order nonlinearity [5], as well as a reduction in film stress. The latter characteristic allows for dramatically simplifying the fabrication process of high confinement waveguides [5]. Recent demonstrations in the silicon-rich silicon nitride platform include broadband wavelength conversion [6], parametric amplification [7] and supercontinuum generation [8]. A significant drawback of silicon-rich silicon nitride is that it comes with an increase in linear absorption loss, typically due to clustering of silicon nanocrystals. Here, we overcome this challenge by finding an optimal composition of silicon-rich silicon nitride that simultaneously provides high confinement, record-low loss $(\sim 0.4$ $\mathrm{dB} / \mathrm{cm}$ ) and high nonlinearity. We demonstrate high-Q microresonators and comb generation in this platform for the first time.
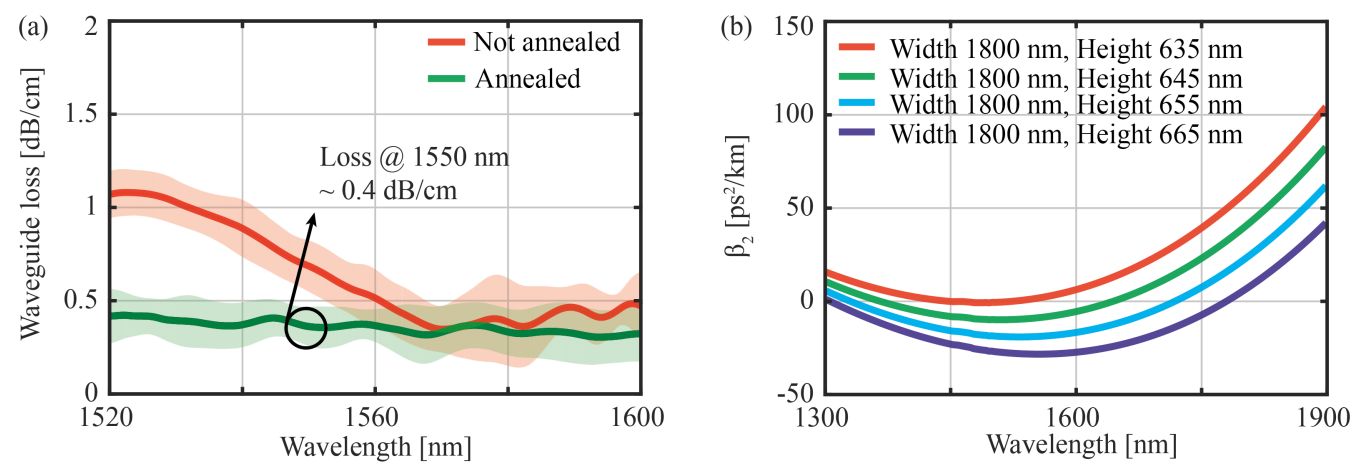

Fig. 1. (a) Measured propagation loss for TE-polarization of straight waveguides, darker colored lines show the mean value and the brighter shadowed areas show the standard deviation of the loss measurement (b) Simulated group velocity dispersion of fundamental TE mode

\section{Waveguide fabrication and characterization}

We fabricated straight waveguides on a $3 \mu \mathrm{m}$ thermally oxidized 3" silicon wafer. Crack-free silicon-rich silicon nitride film with thickness $\sim 645 \mathrm{~nm}$ was deposited in an LPCVD furnace at $770{ }^{\circ} \mathrm{C}$. The stoichiometry of the film was indirectly controlled by the ratio between precursor gases [3]. We set the DCS: $\mathrm{NH}_{3}$ ratio to 3.9, which is above what is typically required for stoichiometric $\mathrm{Si}_{3} \mathrm{~N}_{4}$ [3] but below the value used in our previous results [5]. The refractive index at $1550 \mathrm{~nm}$ is 2.07, indicating that the film is only slightly silicon rich [3]. We followed the process steps presented in [5] to fabricate waveguides. Exposure time of deep UV lithography was modified to achieve waveguides with little shrinkage compared to designed size and less loss. The width and height of the fabricated waveguides are measured to be $1800 \mathrm{~nm}$ and $645 \mathrm{~nm}$. We applied a standard cleaning step and annealing process at $1100{ }^{\circ} \mathrm{C}$ in a nitrogen atmosphere for 3 hours after etching. We used the cut-back method with 3 different lengths to separately measure the propagation loss for TE-polarization of straight waveguides with and without annealing. Averaged propagation loss and standard deviation of over 5 waveguides from same wafer are shown in Fig. 1.(a). The propagation loss of waveguides is $0.4 \pm 0.1 \mathrm{~dB} / \mathrm{cm}$ at $1550 \mathrm{~nm}$. Clearly, the absorption peak at $1530 \mathrm{~nm}$ is successfully mitigated by the annealing process. The absence of increased loss indicates a minimum impact of silicon clustering. The geometry of the waveguides is carefully designed to achieve broadband low anomalous dispersion, as indicated in Fig. 1.(b). We note that fairly flat and anomalous dispersion around $\mathrm{C}$ band is achieved with thinner waveguides 
than what is needed for stoichiometric $\mathrm{Si}_{3} \mathrm{~N}_{4}$. We performed a dual pump measurement to evaluate the nonlinear index $\mathrm{n}_{2}$ of the waveguide after annealing. The evaluated $\mathrm{n}_{2}$ is $\sim 1.5-2$ times larger than $\mathrm{Si}_{3} \mathrm{~N}_{4}$, similar to the value reported in [9] for the similar gas ratio.

\section{Microresonator frequency comb generation}

We also fabricated microring resonators with $\sim 100 \mathrm{GHz}$ free spectral range (FSR) using the fabrication method mentioned above. The width and height of the ring waveguide are measured to be $2000 \mathrm{~nm}$ and $600 \mathrm{~nm}$. Same geometry of bus and ring waveguides is adopted for high-ideality coupling [10], and pulley coupling is introduced in order to achieve stronger coupling. We distinguish resonances from different modes by calculating group index from a FSR and comparing it with our simulations. We only observed resonances corresponding to the TM mode family. This may be due to the formation of an air void during plasma enhanced chemical vapor deposition of $\mathrm{SiO}_{2}$ cladding. The transmission spectrum of TM polarization is shown in Fig. 2(a), and a zoomed in the resonance from the red box is shown in Fig. 2(b). We achieved an intrinsic quality factor $\left(Q_{i}\right) \sim 1$ million for the fundamental TM mode.

(a)

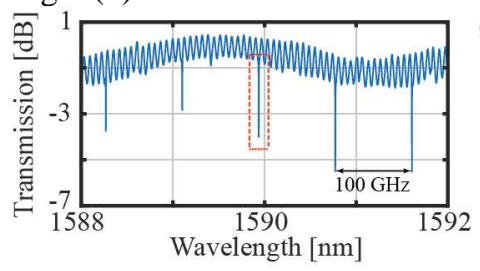

(d)

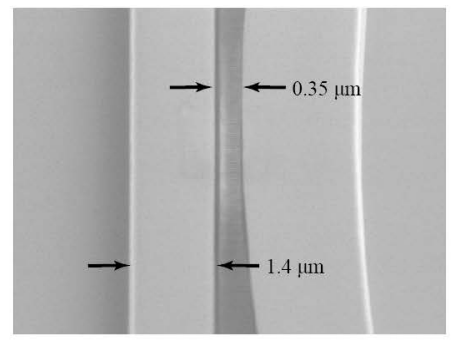

(b)

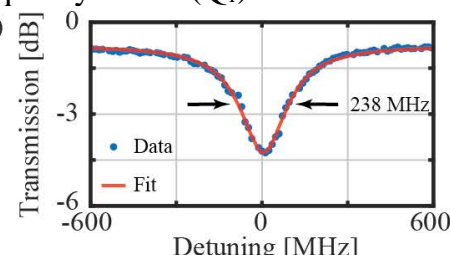

(e)

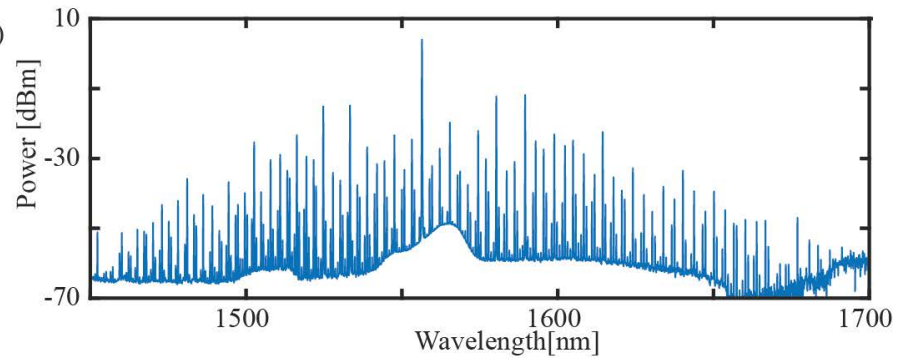

Fig. 2. (a) Normalized transmission spectra of the ring resonator fabricated by deep UV lithography. (b) Finely scanned resonance with 238MHz linewidth. (c-d) Scanning electron microscope (SEM) images of the ring resonator fabricated by EBL. (e) The frequency comb generated from silicon-rich silicon nitride micro-ring resonator

We moved to electron beam lithography (EBL) in order to fabricate ring resonators with smaller gaps to increase the coupling strength. The width and height of the fabricated ring resonator with $100 \mathrm{GHz}$ FSR are $1800 \mathrm{~nm}$ and 655 $\mathrm{nm}$. The SEM images of the ring resonator and its zoomed in gap region are shown in Fig. 2.(c,d). We achieved $\mathrm{Q}_{\mathrm{i}} \sim$ 0.8 million and coupling quality factor $\left(\mathrm{Q}_{\mathrm{c}}\right) \sim 2.2$ million for fundamental TE mode. Although these values are below the recent record results achieved with stoichiometric silicon nitride [2], the complexity in our fabrication process is significantly decreased. We haven't achieved resonators with better $\mathrm{Q}_{\mathrm{i}}$ than the ring resonators fabricated by deep UV lithography so far, but stronger coupling strength is achieved by the possibility of fabricating small gaps ( 350 nm) using EBL. We pumped this device at $1556 \mathrm{~nm}$ with estimated $\sim 160 \mathrm{~mW}$ continuous-wave power coupled in the bus waveguide and obtained the 1-FSR spaced frequency comb shown in Fig. 2.(e).

In summary, by improving lithography steps, controlling the ratio of precursor ratio and annealing temperature, we have been able to fabricate silicon-rich silicon nitride waveguides with low loss $(\sim 0.4 \mathrm{~dB} / \mathrm{cm})$, high-Q microresonators and frequency comb generation. The enhanced nonlinearity and robustness of the fabrication process indicate that silicon-rich silicon nitride is an extremely promising platform for nonlinear optics applications.

\section{References}

[1] J. S. Levy et al., "CMOS-compatible multiple-wavelength oscillator for on-chip optical interconnects," Nat. Photonics 4, 37-40 (2010).

[2] X. Ji et al., "Ultra-low-loss on-chip resonators with sub-milliwatt parametric oscillation threshold," Optica 4, 619 (2017).

[3] T. Makino, "Composition and Structure Control by Source Gas Ratio in LPCVD SiN[sub x]," J. Electrochem. Soc. 130, 450 (1983).

[4] K. K. Oskinen et al., "Enhancement of bulk second-harmonic generation from silicon nitride films ....," Opt. Lett. 42, 18-21 (2017).

[5] C. J. Krückel et al., "Linear and nonlinear characterization of low-stress high-confinement silicon-rich ....," Opt. Express 23, 25827 (2015).

[6] M. R. Dizaji et al., "Silicon-rich nitride waveguides for ultra-broadband nonlinear signal processing," Opt. Express 25, 12100 (2017).

[7] K. J. A. Ooi et al., "Pushing the limits of CMOS optical parametric amplifiers with USRN:Si7N3 ....," Nat. Commun. 8, 13878 (2017).

[8] X. Liu et al., "Octave-spanning supercontinuum generation in a silicon-rich nitride waveguide," Opt. Lett. 41, 2719 (2016).

[9] C. J. Krückel et al., "Optical bandgap engineering in nonlinear silicon nitride waveguides," Opt. Express 25, 770-776 (2017).

[10] M. H. P. Pfeiffer et al., "Coupling Ideality of Integrated Planar High- Q Microresonators," Phys. Rev. Appl. 7, 1-9 (2017).

This work was financially supported by the swedish research council (VR). 